\title{
I-DEAS Finite Element Analysis and Modeling Research
}

\author{
Jinling Wang
}

Shandong vocational college of light industry, Shandong, Zbo, 255300, China.

Keywords: I-DEAS; Finite element analysis; Modeling study; Meshing

\begin{abstract}
In this paper, the I-DEAS software and the finite element method is summarized. Then, the basic process of the finite element analy sis for I-DEAS is described, including the finite element model, the meshing and the boundary conditions. Finally, this paper focuses on the modeling of I-DEAS finite element analysis, including thermal analysis of finite difference method and thermal conduction and heat capacity.
\end{abstract}

\section{Introduction}

Finite element method for a wide range of applications, it cannot only solve the engineering problems of linear and nonlinear for a variety of different properties of solid materials, such as isotropic and anisotropic material, viscoelastic and plastic materials, it can also solve problems of fluid.. In addition, the project in the most universal significance of the unsteady problem also can be addressed. It even can simulate the high-speed collision among components, explosive and stress wave propagation.

\section{I-DEAS software and finite element method}

I-DEAS software overvie w. I-DEAS is the product of SDRC (Dynamics Research Corporation Structural). I-DEAS software is a solution which is fully integrated and is used in the twenty-first century. The complete scheme of perforative is from conception to the design development and production of the whole process, covering the mechanical design automation, product data management, Collaborative Product Commerce and information engineering and implementation of services.

1) Simulation is one of the modules, and the simulation Modeling (Set) is the finite element modeling and visualization tool in the integrated environment of MDA. Direct use of IDeas main software module or assembly, or other CAD systems input model rapid establishment of digital product estimation model, simulation model and design model are relevant.

Solution Linear Simulation is a finite element solver, including linear static analysis and structural modal analysis of the structure.

2) Model solution non - linear supports the geometric nonlinear, material nonlinear, elastic and plastic and synthesis of nonlinear analysis, using Newton Raphson method for solving nonlinear equations.

3) Variable analysis makes the simulation of the design of the initial intervention design process, the use of a single model for a wide range of design and exploration. The variable analysis is automatically solved in the design variable range of the total fluctuating range, and the results of the design manual are generated by a grid division. A variety of comparable schemes are obtained.

Basic concept of finite element method. Many engineering analy sis of the problem, such as solid mechanics in the displacement field and stress field analysis, the vibration characteristics analy sis and heat transfer in the temperature field analysis, fluid mechanics, the flow field analysis can be attributed to under the given boundary conditions in solving the goveming equations for the problem. These control equations can only obtain the analytical solution only in its simple case, and can only be obtained by a numerical method. With the rapid development of computer technology, numerical solution becomes more and more important. The finite element method is a modern computing method 
which is developed rapidly along with the development of electronic computer. The finite element method is best for general purpose and wide application. Its basic idea is to divide the problem solving domain into a series of units, and the nodes are connected only by a node. The amount of the element inside the element can be obtained by the interpolation of the unit node quantity through the selected function relationship. Because of its simple shape element, being easy by the energy or balance relationship established the equation between nodes and each unit equation "set" together and the formation of general algebraic equations, credited to the boundary conditions can be used immediately to solve the equations. The finer the element division is, the more accurate the results are.

Because of the convenience of the finite element program, the accuracy of calculation is high, and the result of calculation has become the reliable basis for the analysis of various indu strial products and design performance.

\section{I-DEAS finite element analysis of the basic process}

\section{Finite element model preparation (Fig. 1)}

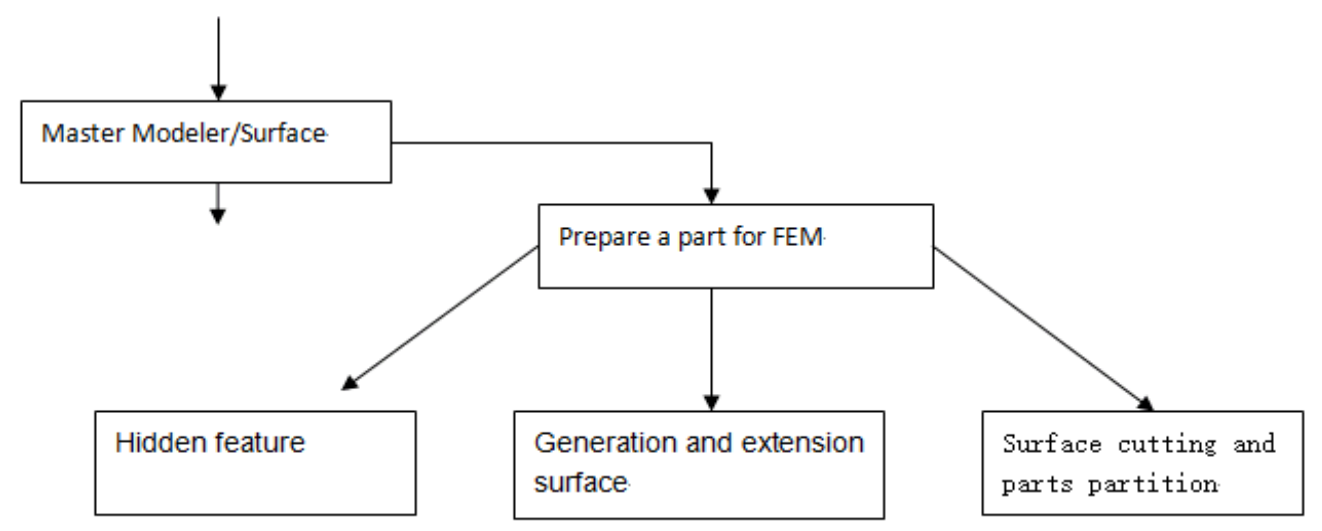

Fig. 1 Model preparation

1) The characteristics of the hidden features are too many, such as chamfer edge, hole-liked, making difficult parts modeling, which can simplify the part to hide unnecessary features. The operation is as follows:

The results of Suppress - > point Update - > point from the point of the menu is taken from the point of the menu to select the parts and to hide the features from the point Modify - >. (Update is used to modify the update model and observe the consequences).

2) Shell surface mesh generation to generation surface - often use the following two methods:

The Surface Plannar is selected. The coplan ar line frame and the ed ge created surface is selected.

By Boundary Surface, the selected line frame and the edge generated surface.

3) When a curve is expected onto the surface, the surface projection curve is useful when defining the curve on the contour surface. The connection between the surface projection and the cutting surface is a typical application. The projection curve has a historical record and can modify its appearance. The curve projection is as follows:

In the plane definition curve - > points Curve Project icon, select the target surface to be expected to the projection of the curve or section - > projection direction.

4) Trimmed camber can be partitioned to define the boundaries of the element or to match the grid. The operation is as follows:

Take surface operators icon, open surface abstraction panel and take trim surface, choose to cut or partition of the surface, selection is utilized to divide the surface curve and surface. 
5) The stitching surfaces can be merged together in common edges and eliminated, and the switching surfaces are as follows:

From the Abstraction Surface Stitch icon to select the Surface Select from the implicit menu selects all Surface.

Meshing. Free mesh: free mesh is generated automatically on the geometry surface and the entity. This grid form is more flexible than the mapping grid, and the boundary of the surface and the entity can be more complex. It can be subdivided into the surface, the body, the hole and the internal cavity

(Fig. 2).

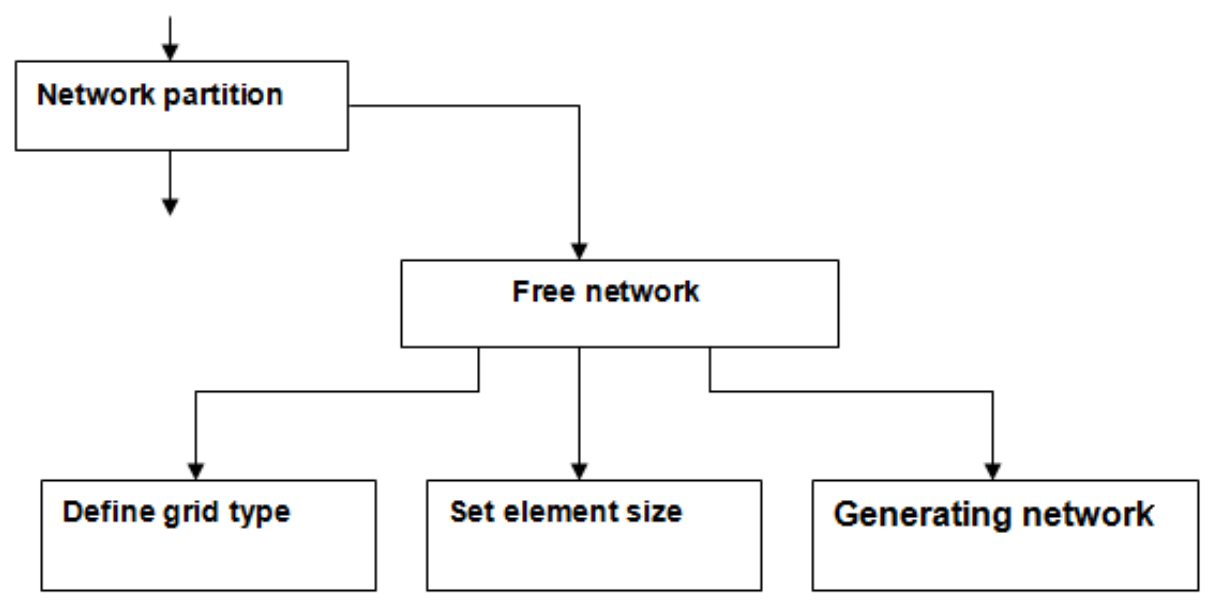

Fig. 2 Generate free mesh

Establish boundary conditions. Basic steps of creating boundary conditions.

1) Select analy sis types, such as Static Linear. Analysis type or command button is equivalent to a filter, once selected, the user will only see the option of the boundary conditions for the analysis type valid. The default analy sis type is Static Linear.

2) Select the appropriate icon menu or command menu to create the required boundary conditions.

3) The entity of the bound ary conditions. The boundary conditions can be loaded into geometric entities such as edges, surfaces, cross-sections, vertices, and arbitrary points on the edges and surfaces, and can also load a finite element entity, such as nodes and units.

4) Add a subset of the bound ary conditions for the collection of the bound ary conditions. Each time the boundary condition is created is filled into a subset of the boundary conditions, which can be selected into a collection of bound ary conditions. Once, a collection of boundary conditions is utilized.

Boundary condition set.

In I-DEAS software, to define the model constraint, degree of freedom coupling, contact, DOF, temperature, and load boundary conditions are preserved in a corresponding group, and call for the corresponding collection. These sets can be divided into a larger set, called boundary conditions, equivalent to one of the condition s of the project. In the set analysis, one or more bound ary conditions can be selected (i.e. one or more working conditions), and each one contains all the boundary conditions for any type of arbitrary type which is suitable for the analysis.

\section{Study on the finite element analysis of I-DEAS}

Finite diffe rence method for thermal analysis. This software uses the control volume method to establish the discretization of the finite difference equation with the partial differential control equation. This method is also called lumped parameter method, which is based on local energy conservation and global energy conservation in the region. This technique consists of geometrical discretization or mesh generation of the mesh into the control volume zone, and FD (finite difference) 
computing points are established within each zone. Determine the heat balance equation for each control volume. For the control volume, the universal thermal equilibrium equation is:

$$
\begin{aligned}
\int_{A} q_{n} \mathrm{~d} a+\int_{V} q_{o} \mathrm{~d} V & =\int_{V} \rho c\left(\frac{\partial T}{\partial t}\right) \mathrm{d} V \\
Q_{b}+Q_{o} & =\left(\frac{\mathrm{d} U}{\mathrm{~d} t}\right)
\end{aligned}
$$

$\mathrm{Qb}$ is the heat flux through controlling the volume boundary; Qo is the heat flux generated within the control volume; $U$ is the energy stored in the volume of control.

This equation is not only applied to a single control volume, but also in the whole problemarea. The heat flux of the boundary between adjacent control volumes can be characterized by heat transfer coefficient G:

$$
Q_{i j}=G_{i j} \times f\left(T_{i}, T_{j}\right)
$$

Temperature $\mathrm{Ti}$ and $\mathrm{Tj}$ are estimated at the control volume of FD. For the linear case of thermal conductivity, $\mathrm{G}$ is constant, and the heat flux is proportional to the temperature of FD. The radiation heat transfer coefficient, the convective heat transfer coefficient and other nonlinear heat transfer coefficients involve more complex temperature functions.

The use of the control volume density and specific heat are supposed to be constant, the heat capacity can be simplified to:

$$
\frac{\mathrm{d} U}{\mathrm{~d} t}=\rho c V \frac{\mathrm{d} T_{i}}{\mathrm{~d} t}=C \frac{\mathrm{d} T_{t}}{\mathrm{~d} t}
$$

Thus, the heat balance equation for controlling volume I is:

$$
Q_{i}+\sum G_{i j} \times f\left(T_{i}, T_{j}\right)=C_{i} \frac{\mathrm{d} T_{i}}{\mathrm{~d} t}
$$

Qi and Ci may be dependent on time and (or) temperature. Under the steady state condition, the equation has no time to the time:

$$
Q_{i}+\sum G_{i j} \times f\left(T_{i}, T_{j}\right)=0
$$

This will generate a series of alg ebraic equations in the problem area, and can be solved by solving the temperature value of the volume FD.

The thermal conduction and heat capacity. The finite difference software can automatically calculate an arbitrary element mesh for heat capacity and heat transfer coefficient. If given a divided grid geometry as well as the unit of material properties, finite difference divided into heat transfer coefficient and the heat capacity matrix can be generated automatically. The calculation unit for heat capacity $\mathrm{Ci}$ :

$$
C_{i}=\rho c V
$$

In the formula, $\mathrm{P}$ unit density; $\mathrm{C}$ unit $\mathrm{V}$ unit volume heat capacity.

The calculation formula for the conduction heat flow between two units adjacent and connected each other is:

$$
Q_{i j}=G_{i j} \times\left(T_{i}-T_{j}\right)
$$

Gij for heat transfer coefficient.

For each unit in the model, I-DEAS is built on its center of mass and its boundaries. The heat transfer between the boundary points is determined by using linear element temperature function of the heat conduction control equation. The centroid calculating point is used for calculating distributed heat transfer: radiation, convection and heat flow. The temperature of the point is calculated by the temperature of the assumed element in the case of a parabolic distribution. The heat flow of the point is assigned to the points on the boundary. 


\section{Conclusions}

The finite element analysis, computer graphics, optimization of reliability technology and combination can form a complete computer aided design system, which can significantly improve the performance of product design, shorten the production cycle to enhance the market competitiveness of products. At present, the application of the finite element method has been used in all fields of mechanical, construction, mining, metallurgy, material, chemical, energy, transportation, electromagnetism and even daily life design and analy sis.

\section{References}

[1] Hongwu Zhang. Finite element analysis and CAE based [M]. Beijing: Tsinghua University press, 2004.

[2] Xucheng Wang, min Shao. Basic principle and numerical method of finite element method [M]. Tsinghua University press, 1997.

[3] Hong Ye.I-DEAS thermal analysis practical tutorial [M]. China University of technology and Technology University Press, 2003.

[4] Ridong Liao. Tutorial: finite element analy sis [M]. Beijing Institute of Technology press, 2003.

[5] Deren Xie. Thermal design of electronic equipment [M]. Southeast University press, 1989:189-194.

[6] Jun Xue, Baoyu Sun. Application of thermal analysis technology in the thermal design of electronic equipment [J]. Chan gchun University of Technology press, 2007, (2), 176-179.

[7] R.D. Cook. The concept and application of finite element analysis [M]. Beijing Science Press, 1989.

[8] Ren Wang, Wenbin Huang. The plastic mechanics theory [M]. Peking University press, 1982.

[9] Lu Zhou, Fuqun Fan. Mechanics of composite materials [M]. Beijing: Higher Education Press, 1991.

[10] You liang Jiang.nonlinear finite element method [M]. Beijing In stitu te of industry, 1988. 